\title{
PARKER INSTABILITY AND THE GALACTIC DYNAMO
}

\author{
M. HANASZ* and H. LESCH \\ Max-Planck-Institut für Radioastronomie, Auf dem Hügel 69, W-5300 Bonn, FRG
}

\section{The model of interstellar medium}

We imagine the interstellar medium as a system of molecular clouds and isolated magnetic flux tubes. We assume that: the flux tubes appear as a result of disruption of molecular clouds containing magnetic field, their length is comparable to the mean intercloud distance of about $100 \mathrm{pc}$ and diameter comparable to the diameter of the clouds $\sim 10 \mathrm{pc}$.

\section{The dynamics of isolated flux tubes}

It was demonstrated by Parker (1967 and references therein) that the composition of ionized gas, magnetic field and cosmic radiation, placed in a gravitational field is unstable against buoyancy instability, referred to as Parker instability.

We apply the thin tube approximation (see eg. Spruit 1981) for a slender, initially horizontal flux tubes embedded in a stratified external (e) atmosphere with pressure $p_{e}(z)$, density $\rho_{e}(z)$ and vanishing magnetic field. The internal (i) gas pressure is $\mathrm{p}_{\mathrm{i}}$ and density is $\rho_{\mathrm{i}}$. We consider dynamics of the flux tubes balancing the competing buoyancy under the galactic gravitational acceleration $\mathrm{g}$ (in the negative z-direction) and aerodynamic drag forces. Equations of hydrostatic equilibrium $p_{e}(z)=p_{i}(z)+\frac{B(z)^{2}}{8 \pi}$, isothermal evolution $p_{i}(z)=v_{s}^{2} \rho_{i}(z)$, where $v_{s}^{2}=\frac{k_{B} T}{m_{p}}$ is the isothermal sound speed and conservation of magnetic flux inside the tube $B(z) a(z)^{2}=B_{0} a_{0}^{2}$, determine the evolution of radius of the tube with height: $a(z) / a_{0}=\exp (z / 4 H)$. Balance of the buoyancy force per unit length of the flux tube $F_{B}=-\pi g\left(\rho_{i}(z)-\rho_{e}(z)\right) a(z)^{2}$ and the aerodynamic drag force (Schüssler, 1977) $F_{D}=\rho_{e}(z) v_{z}^{2} a(z) C_{D}$ with the drag coefficient $C_{D} \sim 1$ gives the vertical speed of the order of only $1 \mathrm{kms}^{-1}$. The Coriolis acceleration of the gas sinking along the tube allows to estimate the mean value of the cyclonic velocity after the characteristic time of rise (see Hanasz \& Lesch 1993).

$$
<v_{\varphi}>\simeq 0.5 \Omega L \simeq 1.5 \mathrm{kms}^{-1}\left[\frac{L}{100 \mathrm{pc}}\right]\left[\frac{\Omega}{10^{-15} \mathrm{~s}^{-1}}\right] \text {. }
$$

\section{The helicity and diffusivity of the system of buoyant flux tubes}

Applying the formula derived by Parker (1979) for the $\alpha$-coefficient in the dynamo equation $\alpha \simeq 0.4\left\langle v_{\varphi}\right\rangle$, we obtain for our model

$$
\alpha \sim 0.6 \mathrm{kms}^{-1}\left[\frac{v_{\varphi}}{1.5 \mathrm{kms}^{-1}}\right] .
$$
$12 / 18$

* On leave from: Institute of Astronomy, N. Copernicus University 87-100 Torun, ul. Chopina 
We assume that to the accuracy of a factor of order of 1 the numerical coefficient 0.4 should be comparable in the geometries of the convective cell (considered by Parker) and the flux tube. Similarly, for the turbulent diffusivity we apply $\eta_{T} \sim 0.2 v_{T} l_{T}$, where $l_{T} \sim L \sim 100 \mathrm{pc}$ is the dominant scale of the turbulent eddies and $v_{T}$ is the turbulent velocity. Concerning $v_{T}$ we have to distinguish the two following components of motion of the interstellar matter in the frame of our model: the chaotic motions of molecular clouds (that do not participate in the $\alpha$-effect, but participate in the turbulent diffusion) and the buoyant motions of flux tubes that participate in both the $\alpha$-effect and diffusion. The diffusivity coefficient calculated for the pure first component with $v_{T} \sim v_{\text {cloud }} \sim 10 \mathrm{kms}^{-1}$ is

$$
\eta_{T}=0.6 \cdot 10^{26} \mathrm{~cm}^{2} \mathrm{~s}^{-1}
$$

For the pure second component with $v_{T} \sim v_{z} \sim 1 \mathrm{kms}^{-1}$ we obtain

$$
\eta_{T}=0.06 \cdot 10^{26} \mathrm{~cm}^{2} \mathrm{~s}^{-1}
$$

It seems therefore that the second component taken alone gives small contribution to diffusivity but on the other hand it is possible that the diffusion of magnetic field is related to the slow flux tube velocities $\sim 1 \mathrm{kms}^{-1}$. It can be simply imagined that the mesh of braked flux tubes moving through the surrounding gas cannot reach the speeds higher then the above value, but the mesh can be elastic enough to allow individual clouds reach temporal speeds up to $10 \mathrm{kms}^{-1}$.

\section{Conclusions}

The value of $\alpha \sim 0.6 \mathrm{kms}^{-1}$ is in order of magnitude comparable to that obtained in other estimations of this quantity (see e.g. Ferriere 1993).

We showed that due to the aerodynamic drag force acting on the flux tube $\eta_{T}$ can be decreased by the factor of 10 below the standard value $\eta_{T} \sim 0.6 \cdot 10^{26} \mathrm{~cm}^{2} \mathrm{~s}^{-1}$. In another words the aerodynamic drag protects the magnetic field against the fast escape from the galactic disc. This causes that both the magnetic Reynolds numbers $R_{\alpha}=\frac{\alpha H}{\eta_{T}}$ and $R_{\omega}=\frac{r \frac{\partial \Omega}{\partial r} H^{2}}{\eta_{T}}$ increase by a factor of 10 and in consequence the dynamo number $D=R_{\alpha} R_{\omega}^{\eta_{T}}$ can be as large as a few hundreds in the present model.

M.H. is very grateful to the organizers of the Symposium and the I.A.U for financial support to the participation at the Symposium.

\section{References}

Ferriere, K.: 1993, This proceeding

Hanasz, M., Lesch, H.: Submited to Astron. Astrophys.

Parker, E.N.: 1967b, Ap.J. 149, 535

Parker, E.N. 1979, Cosmical Magnetic Fields, Clarendon Press, Oxford.

Schüssler, M.: 1977, Astron. Astrophys. 56, 439

Spruit, H.C.: 1981, Astron. Astrophys. 98, 155 